\title{
Nanoscale sliding friction versus commensuration ratio: Molecular dynamics simulations
}

\author{
Evy Salcedo Torres, ${ }^{1, *}$ Sebastián Gonçalves, ${ }^{1,2, \dagger}$ Claudio Scherer, ${ }^{1, \$}$ and Miguel Kiwi ${ }^{3, \S}$ \\ ${ }^{1}$ Instituto de Física, Universidade Federal do Rio Grande do Sul, Caixa Postal 15051, 91501-970 Porto Alegre RS, Brazil \\ ${ }^{2}$ Consortium of the Americas for Interdisciplinary Science and Department of Physics and Astronomy, \\ University of New Mexico, Albuquerque, New Mexico 87131, USA \\ ${ }^{3}$ Facultad de Física, Pontificia Universidad Católica, Casilla 306, Santiago, Chile 6904411
}

(Received 30 September 2005; revised manuscript received 30 November 2005; published 30 January 2006)

\begin{abstract}
The pioneer work of Krim and Widom [Phys. Rev. B 38, 12184 (1988)] unveiled the origin of the viscous nature of friction at the atomic scale. This generated extensive experimental and theoretical activity. However, fundamental questions remain open like the relation between sliding friction and the topology of the substrate, as well as the dependence on the temperature of the contact surface. Here we present results, obtained using molecular dynamics, for the phononic friction coefficient $\left(\eta_{p h}\right)$ for a one-dimensional model of an adsorbatesubstrate interface. Different commensuration relations between adsorbate and substrate are investigated as well as the temperature dependence of $\eta_{p h}$. In all the cases we studied $\eta_{p h}$ depends quadratically on the substrate corrugation amplitude, but is a nontrivial function of the commensuration ratio between substrate and adsorbate. The most striking result is a deep and wide region of small values of $\eta_{p h}$ for substrate-adsorbate commensuration ratios between $\approx 0.65$ and 0.9 . Our results shed some light on contradictory results for the relative size of phononic and electronic friction found in the literature.
\end{abstract}

DOI: 10.1103/PhysRevB.73.035434

PACS number(s): 68.43.-h, 81.40.Pq, 46.55.+d

\section{INTRODUCTION}

The pioneer work of Krim and Widom, revealing the viscous nature of the friction of a krypton monolayer sliding over a gold substrate, ${ }^{1}$ generated a flurry of theoretical work intended to develop an understanding of this interesting phenomenon..$^{2-5}$ Subsequently, a huge body of new and fascinating experiments on nanoscale friction has seen the light during the last 15 years. However, the theoretical interpretation of nanoscopic sliding experiments has evidenced some degree of disagreement, mainly related to incompatible results between different simulations, ${ }^{3-5}$ in spite of the fact that they were performed for the same system with similar models and techniques. With the aim of finding some plausible explanation for these discrepancies we set out to study the relation between the topology and sliding friction coefficient $\eta$. Specifically, we conduct a careful study of a onedimensional model to disclose the relation between sliding atomic friction and the substrate-adsorbate commensuration ratio.

Understanding the origin of sliding friction is a fascinating and challenging enterprise. ${ }^{6,7}$ Issues like how the energy dissipates on the substrate, which is the main dissipation channel (electronic or phononic), and how the phononic sliding friction coefficient depends on the corrugation amplitude were addressed, and partially solved, by several groups. ${ }^{2-5}$ Yet in the specific case of Xe over Ag, three different groups tried either to explain or to estimate theoretically the experimental observations of Daily and Krim. ${ }^{8}$ Despite the fact that they used similar models and similar simulation techniques to calculate the phononic contribution to friction, they got quite different results. Persson and Nitzan $^{3}$ found that the phononic friction was not significant in comparison with the electronic contribution, while Tomassone et $a l .{ }^{4}$ found the opposite. On the other hand Liebsch et al. ${ }^{5}$ concluded that both contributions are important, but that the phononic fric- tion strongly depends on the substrate corrugation amplitude. The abrupt change in the sliding friction at the superconductor transition observed by Dayo et al. ${ }^{9}$ provides additional support to the latter argument, showing that the electronic friction is of the same order of magnitude as the phononic one.

However, the relation between substrate corrugation and phononic friction cannot explain the full magnitude of the disagreement between the different authors. To achieve full agreement we would be forced to allow for huge differences in the corrugation amplitude between the various models, much larger than in actual fact. An apparently subtle technical detail or artifact of the molecular dynamics simulation might show the path to the answer of such divergences, either by itself or by clarifying and improving on the abovementioned corrugation dependence.

When carrying out molecular dynamics simulation the Xe adsorbate adopts one of two different orientations relative to the $110 \mathrm{Ag}$ substrate. This in turn produces significant changes in the effective sliding friction coefficient, which maybe due to the different commensuration ratios between substrate and adsorbate, since the adsorbate adopts one or the other of the two preferred orientations. With the objective of elucidating this question here we present molecular dynamics results for a one-dimensional system. Our aim is to stress the role, and at the same time to have complete control of, the relation between $\eta$ and the commensuration ratio.

\section{MODEL}

The model, schematically depicted in Fig. 1, can be thought of as a generalized Frenkel-Kontorova model plus a fluctuation-dissipation mechanism. It consists of a onedimensional chain of atoms that interact with each other through a Lennard-Jones interatomic potential (adsorbate), 


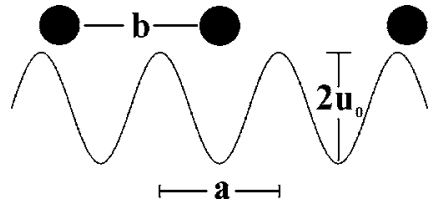

FIG. 1. The one-dimensional model.

moving in a periodic external potential (substrate). Apart from the interatomic and adsorbate-substrate interactions, there are damping and stochastic forces that act as a thermostat, plus an external applied force which makes the adsorbate slide over the substrate. This way, we intend to model the sliding of a solid monolayer over a perfect crystalline substrate. Therefore, the adatoms of mass $m$ and labeled by the indices $i$ and $j$ obey the following Langevin equation:

$$
m \ddot{x}_{i}+m \eta_{e} \dot{x}_{i}=-\sum_{j} \frac{\partial V\left(\left|x_{i}-x_{j}\right|\right)}{\partial x_{i}}-\frac{\partial U\left(x_{i}\right)}{\partial x_{i}}+f_{i}+F .
$$

The external force $F$ is applied to every atom in the chainthe total external force is thus $N F$-and $f_{i}$ is a stochastic fluctuating force drawn from a Gaussian distribution and related to $\eta_{e}$ via the fluctuation-dissipation theorem

$$
\left\langle f_{i}(t) f_{j}(0)\right\rangle=2 \eta_{e} m k_{B} T \delta_{i j} \delta(t),
$$

where $T$ is the temperature of the substrate and $k_{B}$ is the Boltzmann constant. The stochastic force plus the dissipation term provides a thermal bath to describe the otherwise frozen substrate. Moreover, the damping term represents the electronic part of the microscopic friction, which cannot be included in a first-principles way in our classical treatment. $\eta_{e}$ may thus be regarded as the electronic sliding friction coefficient. The expression of the interatomic Lennard-Jones potential between adatoms, $V(r)$, where $r=\left|x_{i}-x_{j}\right|$, is given by

$$
V(r)=\varepsilon\left[\left(\frac{r_{0}}{r}\right)^{12}-2\left(\frac{r_{0}}{r}\right)^{6}\right],
$$

where $r_{0}$ is the $T=0$ equilibrium distance of the dimer and $\varepsilon$ is the depth of the potential well. The interaction is cut off beyond third neighbors, and hence the cell parameter for the isolated chain is $b=0.9972 r_{0}$. The adsorbate-substrate potential $U(x)$ is a periodic potential

$$
U(x)=u_{0}\left[\cos \left(2 \pi \frac{x}{a}\right)+1\right]
$$

and therefore $a$ is the periodicity of the potential, representing the distance between neighboring substrate atoms; $u_{0}$ is the semiamplitude of the adsorbate-substrate potential, and it is usually called the substrate corrugation.

In what follows we take $r_{0}, \varepsilon$, and the mass $m$ of the adsorbate atoms as the fundamental units of the problem, expressing all other quantities in terms of them. For example, the time unit is $t_{0}=r_{0} \sqrt{\mathrm{m} / \varepsilon}$ and temperatures, presented as $k_{B} T$, are in units of $\varepsilon$.

The periodic boundary conditions impose the following relation between $a$ and $b$ :

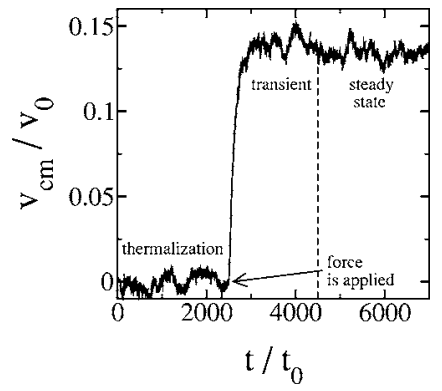

FIG. 2. Typical behavior of the adatom center-of-mass velocity $\left(v_{\text {c.m. }}\right)$ during a Langevin molecular dynamics run, before and after the force is applied. The temperature is $k_{B} T=0.05 \varepsilon$, and the applied force is $F=10^{-3} \varepsilon / r_{0}$; in this case, $a / b=0.95$ and $u_{0}=0.04 \varepsilon \cdot v_{0}=\sqrt{\varepsilon / m}$ and $t_{0}=r_{0} \sqrt{m / \varepsilon}$.

$$
N_{s} a=N_{a} b,
$$

where $N_{s}$ is the number of substrate atoms and $N_{a}$ the number of adsorbed atoms. Therefore, the commensuration ratio between substrate and adsorbate is given by

$$
\frac{a}{b}=\frac{N_{a}}{N_{s}} .
$$

As was stated in Sec. I, several authors ${ }^{2-5}$ have already stressed the important role of coverage and corrugation in the understanding of atomic sliding friction. Here we focus on the effect of these parameters, restricting the system to be one dimensional, in order to avoid any possible topological artifacts that are due to finite-size effects. Consequently $a / b$ and $u_{0}$ are our key parameters in the study of the adsorbatesubstrate interface response to sliding friction.

Equation (1) is numerically integrated using a Langevin molecular dynamics algorithm ${ }^{10-12}$ for 2500 particles $\left(N_{a}\right)$, with a time step $\Delta t=0.01 t_{0}$. To obtain the friction coefficient $\eta$ an external force $F$ is applied; however, before this force is applied we allow the system to relax during $t_{r}=2500 t_{0}$. Next, $F$ is applied to every adatom, and after another transient period of the same extension the adatoms are presumed to have reached the steady state, where the average center-ofmass velocity is $v_{\text {c.m. }}$. At that stage the average friction force equals the external force (this conjecture is shown below to be valid),

$$
F=m \eta v_{\text {c.m. }},
$$

and hence $\eta$ is the effective microscopic friction coefficient which includes the ad hoc friction coefficient $\eta_{e}$ of Eq. (1). Except when noted, in all calculations we keep the electronic contribution to $\eta$ fixed at $\eta_{e}=5 \times 10^{-3} t_{0}^{-1}$. We will see in Sec. III B that it is possible to assume that the effective friction coefficient is $\eta=\eta_{e}+\eta_{p h}$; therefore, the precise value of $\eta_{e}$ should be irrelevant for the calculation of $\eta_{p h}$. We will comment on the validity of this assumption in Sec. III D.

Figure 2 shows the typical behavior of the adatom centerof-mass velocity during a Langevin molecular dynamics run, before and after the external force is applied. We observe that the system relaxes in a typical time which is less than $1000 t_{0}$ during the thermalization period and that it enters into a 

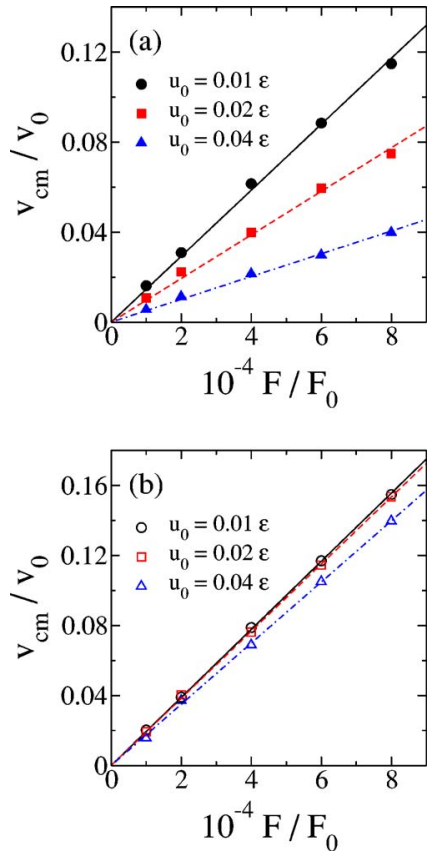

FIG. 3. (Color online) Center-of-mass velocity $v_{\text {c.m. }}$ as a function of the external force $F$ for different substrate corrugation values and for two commensuration ratios: (a) $a / b=0.96$ and (b) $a / b$ $=0.6$, at temperature $k_{B} T=0.1 \varepsilon$. The resulting $\eta$ coefficients are $0.0068(\bullet), 0.010(\square), 0.0198(\mathbf{\Delta}), 0.0051(\square), 0.0052(\square)$, and $0.0057(\triangle)$, all in units of $\omega_{0}=\sqrt{\varepsilon /\left(m r_{0}^{2}\right)} . F_{0}=\epsilon / r_{0}$.

steady state in about the same time after the force is applied. The applied force is $F=0.001 \varepsilon / r_{0}$, and the asymptotic timeaveraged center of mass velocity $v_{\text {c.m. }}=0.133 r_{0} / t_{0}$; therefore, the resulting friction coefficient is $\eta=0.0075 t_{0}^{-1}$, which implies $\eta_{p h}=0.0025 t_{0}^{-1}$.

\section{RESULTS}

As was stated above, in this contribution our main concern is the relation between atomic sliding friction and adsorbate-substrate interface topology. Consequently, in this section we present results for our model of the effective sliding friction coefficient $\eta$, for different values of the substrate-adsorbate commensuration ratio $a / b$, and of the substrate corrugation $u_{0}$. We also check on the effect of substrate temperature.

\section{A. Force-velocity relation}

Once we have made sure that the atomic friction obeys Eq. (7), we obtain from it the effective friction coefficient $\eta$ simply by evaluating the slope of the $F \propto v$ relation. The force $F$ is the input value, and the velocity is obtained by time averaging the steady-state center-of mass-velocity, as exemplified for one run in Fig. 2. To obtain reliable data we perform five independent runs for each value of $F$ and we take the average of the resulting $v_{\text {c.m. }}$ values. A set of results for $a / b=0.96$ and $a / b=0.6$ is presented in Fig. 3, where it is observed that, although some small deviations are present in the first case, the linear relation between $v_{\text {c.m. }}$ and $F$ is a valid
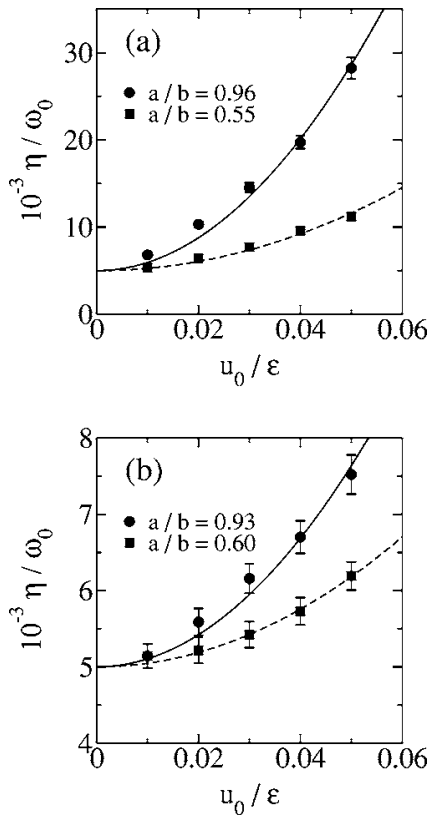

FIG. 4. Total friction coefficient $\left[\omega_{0}=\sqrt{\varepsilon /\left(m r_{0}^{2}\right)}\right]$ for some commensuration ratios $a / b$ and for a temperature $k_{B} T=0.1 \varepsilon$ : (a) "strong"-friction coefficients, the lines are fittings to $\eta_{e}+9.45 u_{0}^{2}$ (solid line) and $\eta_{e}+2.64 u_{0}^{2}$ (dashed line); (b) "weak"-friction coefficients, the lines are fittings to $\eta_{e}+1.05 u_{0}^{2}$ (solid line) and $\eta_{e}$ $+0.47 u_{0}^{2}$ (dashed line).

assumption for all the substrate corrugation values considered here, and this holds for other commensuration ratios. Besides, it is quite evident that the set of results of Fig. 3(a) $(a / b=0.96)$ corresponds to sliding friction that is much more strongly dependent on substrate corrugation $u_{0}$ than the set of Fig. 3(b) $(a / b=0.6)$. This is a remarkable result of our contribution, and it will be discussed in more detail in the next sections.

\section{B. Sliding coefficient vs corrugation}

In Fig. 4 we present the results for the effective friction coefficient at $k_{B} T=0.1 \varepsilon(\approx 22 \mathrm{~K}$ if using Xe parameters) and for different commensuration ratios. In view of what was shown in the previous figure, we present them separated into two plots, depending on the range of variation of $\eta$ as a function of the corrugation amplitude. The cases where the coefficient $\eta$ rises above $9 \times 10^{-3} t_{0}^{-1}$ at the maximum corrugation amplitude $\left(u_{0}=0.05 \varepsilon\right)$ are shown in Fig. 4(a), while the cases with friction coefficient below that value (at the same corrugation amplitude) are displayed in Fig. 4(b). We denominate them, respectively, "strong"- and "weak"friction cases.

Given the theoretical arguments presented by Smith et al. ${ }^{2}$ and the numerical evidence also presented by them and by Liebsch et al. $^{5}$ supporting the quadratic dependence of the friction coefficient with substrate corrugation, we fitted the data of Fig. 4 with the following expression:

$$
\eta=\eta_{0}+c u_{0}^{2}
$$

where $\eta_{0}$ is the friction coefficient in the absence of corru- 

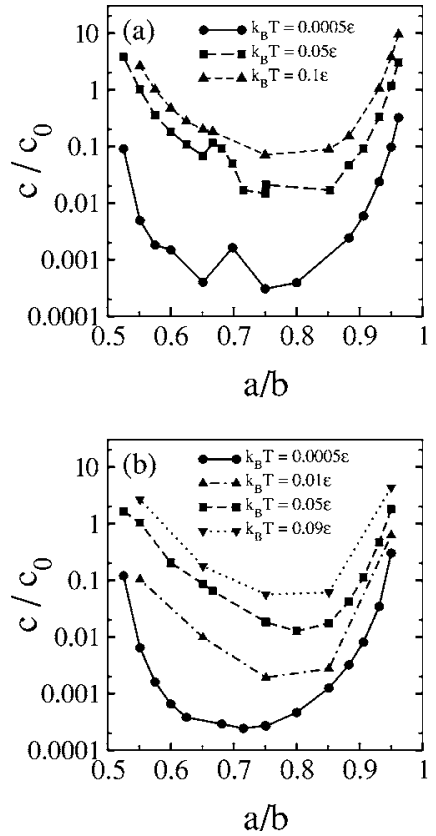

FIG. 5. Coefficient $c$ of the phononic friction $\left(\eta_{p h}=c u_{0}^{2}\right)$ for several commensuration ratios. (a) Results obtained with external force $F$ in the range $(0.0001-0.001) \varepsilon / r_{0}$ and three different temperatures. (b) Results obtained with external force $F$ in the range $(0.001-0.002) \varepsilon / r_{0}$ and four different temperatures. $c_{0}$ $=\left(m r_{0}^{2} \varepsilon^{3}\right)^{-1 / 2}$.

gation and thus equal to the ad hoc (or electronic) friction coefficient $\eta_{e}$. The second term of the effective friction coefficient $c u_{0}^{2}$ is referred to as the phononic friction coefficient $\eta_{p h}$, which depends quadratically on the corrugation amplitude $u_{0}$. The resulting parabolas can be seen in Fig. 4 along with the data. Notice that our results are consistent with Eq. (8) and that $\eta_{0}$ is indeed equal to $\eta_{e}$.

\section{Sliding coefficient vs commensuration}

Once it is properly established that the sliding friction of adsorbate-substrate interfaces due to phonons depends quadratically on the substrate corrugation amplitude, the coefficient $c$ becomes the main source of information about the phononic friction between different surfaces. A synthesis of the results of the present contribution is given in Fig. 5, where we illustrate the behavior of the coefficient $c$ of Eq. (8) over the whole range of commensuration ratios, and for several different temperatures, using a semilogarithmic plot. The principal feature of this figure is the region of low $\eta_{p h}$ values for $a / b \approx 0.65-0.90$. Figures 5(a) and 5(b) come from different ranges of forces. Figure 5(a) was obtained using forces in the range $(0.0001-0.001) \varepsilon / r_{0},{ }^{17}$ while for Fig. 5(b) we used forces in the range $(0.001-0.002) \varepsilon / r_{0}$. In spite of the different ranges of forces used for each set, the two plots are fairly consistent and important conclusions can be drawn from there. The phononic friction changes dramatically as a function of $a / b$, with significantly larger values in the vicinity of low commensuration ratios than far from them. Indeed, at temperatures close to zero the windows for phononic sliding friction are below $10 \%$ of the low order commensuration

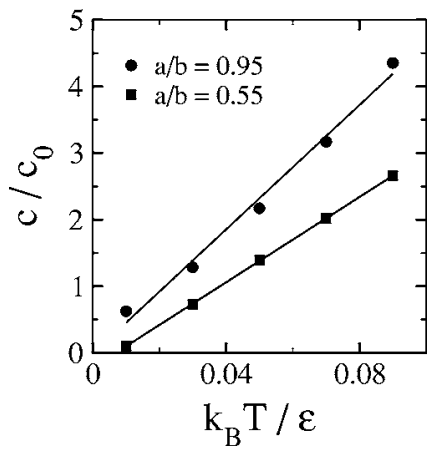

FIG. 6. Temperature dependence of the coefficient $c$ for two values of the commensuration ratio $a / b$, corresponding to the "strong"-friction cases. $c_{0}=\left(m r_{0}^{2} \varepsilon^{3}\right)^{-1 / 2}$.

ratio value [Figs. 5(a) and 5(b), solid line]; such narrow windows become wider and less abrupt when the temperature is increased. Inside the high-phononic-friction windows, such friction depends strongly on the corrugation amplitude, so it can be lower or higher than the electronic friction depending upon the system (values of electronic friction and corrugation amplitude of the specific interface). The small differences between Figs. 5(a) and 5(b) point out the nonlinearity of the friction as the force increases, which will be further discussed in the next section.

We conclude from Fig. 5 that the coefficient $c$ has a nontrivial relation with the commensuration ratio, which allows us to discriminate between strong and weak phononic friction-i.e., as a function of interface mismatch. Besides, it reflects how the friction coefficient varies with temperature; to better illustrate the latter behavior, we present in Fig. 6 the relation between $c$ and temperature for two ("strong" cases) values of the commensuration ratio, extracted from Fig. 5(a). We see that for a fixed $a / b$ ratio the $c$ coefficient increases linearly with temperature. For a commensuration ratio in the "weak" region like $a / b=0.75$ the behavior with temperature is much more dramatic, changing in almost three orders of magnitude when the temperature raises to $k_{B} T=0.1 \varepsilon(\approx 22 \mathrm{~K}$ for $\mathrm{Xe})$.

\section{Linear regime}

The linearity issue is a central concern of our contribution. All the results presented here are obtained from the slope of the $F$-vs- $v$ relation, thus assuming that the system is in the linear regime. However, as linearity is not guaranteed (i.e., one cannot be sure if the slope obtained at some finite range of forces would be the same as $F \rightarrow 0$ ), we provide additional evidence of linearity using an equilibrium fluctuation method. More precisely, we obtain the slip time from the temporal decay of the center-of-mass velocity autocorrelation function. The resulting plots are straight lines in semilogarithmic scale, which is the definite proof of linearity, the inverse of the slope being the sliding coefficient. In Fig. 7 we present $\eta$-vs- $u_{0}$ plots, obtained by both methods: the equilibrium fluctuations procedure described above and the steadystate method used for the bulk of our results. The commensuration ratio is $a / b=0.55$, close to the left border of the 


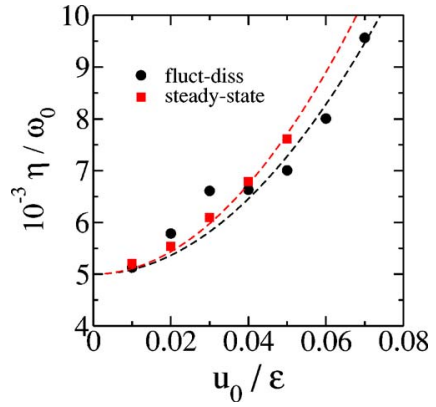

FIG. 7. (Color online) Comparison of methods for obtaining the total friction coefficient, represented as a function of corrugation $u_{0}$ : circles are results from the fluctuation-dissipation method, squares are from the steady-state method, and the lines are quadratic fittings. $k_{B} T=0.05 \varepsilon, a / b=0.55$, and $\omega_{0}=\sqrt{\varepsilon /\left(m r_{0}^{2}\right)}$.

$c$-vs- $a / b$ plot illustrated in Fig. 5 and thus representative of the strong-friction cases. One clearly sees that both methods are consistent, although the $F$-vs- $v$ method has a slight tendency to overestimate the coefficients. Differences between both methods are around $15 \%$, but as our general concern is on investigating the effect of topology, and not modeling a specific system, they are immaterial. Additionally, for $a / b$ values in the central region and at very low temperature, the equilibrium fluctuation method yields sliding coefficients that cannot be distinguished from the ad hoc electronic value, which is perfectly consistent with Fig. 5 . Yet it is possible that the vanishingly small values of the phononic sliding friction in that region could be due to our choice of $\eta_{e}$, which is rather arbitrary. Actually, the value of $\eta_{e}$ has to be inferred by other means and for each specific system. Nevertheless, all in all this gives a rational support for our results and at the same time they do set limits on the validity of the linear regime.

Regarding relation (8), $\eta=\eta_{e}+\eta_{p h}$, supposedly independent of the actual value of $\eta_{e}$, this should be true if $\eta_{e}$ $<1 / t_{p h}$ ( $t_{p h}$ is the intrinsic phonon lifetime at equilibrium). We have checked the robustness of that expression using different values of $\eta_{e}$ up to $\eta_{e} \approx 0.03 t_{0}^{-1}$; the value of $\eta_{p h}$ is constant, provided that $\eta_{e}<0.015 t_{0}^{-1}$. The value we have used throughout our simulations is 3 times smaller.

\section{E. Phonon density of states}

In order to obtain some insight into the origin of the sliding friction "wide minimum" as a function of commensuration ratio, we look at the adsorbate phonon density of states $\mathcal{D}(\omega)$, which can be readily computed, in a molecular dynamics simulation, by means of the Fourier transform of the velocity autocorrelation function $\gamma$, given by ${ }^{13}$

$$
\gamma(t)=\frac{\sum v_{i}(t) v_{i}(0)}{\sum v_{i}(0)^{2}} .
$$

The resulting $\mathcal{D}(\omega)$ spectra are shown in Fig. 8 for one representative $a / b$ ratio of each group, both at the low temperature $T=0.005$; Fig. 8(a) corresponds to the weak- and Fig. 8 (b) to the strong-friction group. We do so for the $F \neq 0$
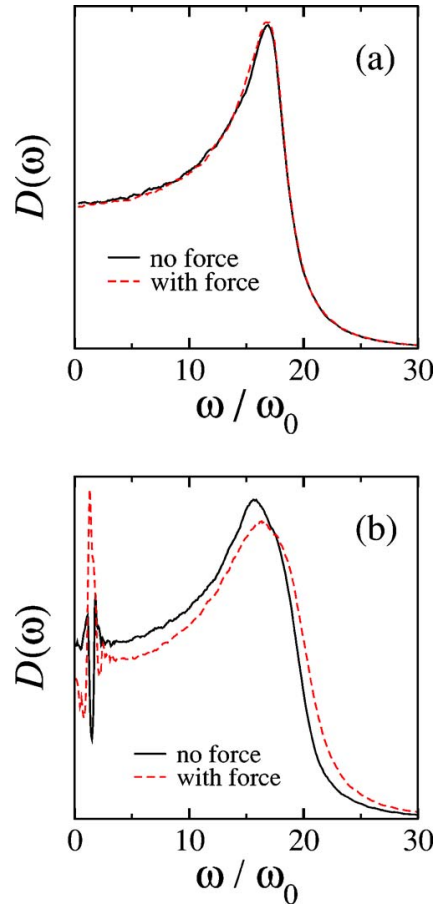

FIG. 8. (Color online) Normalized phonon-frequency spectrum for two different type of commensuration ratio values, one of the (a) weak-friction type $(a / b=0.85)$ and the other of the (b) strongfriction type $(a / b=0.95)$. In both cases the corrugation amplitude is $u_{0}=0.04 \varepsilon$ and the temperature is $T=0.05 \varepsilon / k_{B}$. The frequency $\omega$ is presented in units of $\omega_{0}=\sqrt{\varepsilon /\left(m r_{0}^{2}\right)}$.

(dashed line) and $F=0$ (solid line) steady-state cases, where $F$ is the external applied force. Notice that for the strongfriction case, extrema of $\mathcal{D}(\omega)$ in the low-frequency region are generated, which closely resemble one-dimensional Van Hove singularities-i.e., points which correspond to extrema (maxima or minima) of the $\omega$ versus $k$ dispersion relation. In our model they are due to the mismatch of the chain and periodic potential periodicities. When the external force is applied an inversion occurs: in the frequency region where there is a minimum for the $F=0$ preforce situation, a maximum of $\mathcal{D}(\omega)$ develops during chain sliding. This feature is absent in the weak-friction case, where the phonon density of states is much like the typical one for an isolated onedimensional (1D) system, with no difference between the force-free $(F=0)$ and driven $(F \neq 0)$ cases. As the commensuration ratio $a / b$ crosses over from the strong- to the weakfriction regime the maxima are quenched.

The physical implications of the $\mathcal{D}(\omega)$ maximum are that in the neighborhood of the commensuration values of the $a / b$ ratio (i.e., $a / b \approx 0.5$ or $a / b \approx 1$ ), some low-frequency (long-wavelength) modes cannot be excited, since the adsorbate has to adjust to the mismatch condition. However, when the system starts its sliding motion, precisely these "soft" modes are the ones that are excited preferentially, with the consequent amplitude increase in that frequency region. It is just this feature that singles out the most efficient energy dissipation channel. The way in which this is achieved is by pumping center-of-mass energy into the adsorbate vibration modes that lie in the vicinity of the Van Hove-like singular- 
ity, which increases the friction relative to the weak-friction case, where the mismatch does not impose on the system a frequency region where it does not vibrate "comfortably." Therefore, in the weak-friction regime, the phononic dissipation channel is just the usual one.

To provide additional support to this interpretation we plot, in Fig. 9, "snapshots" of the system as it evolves in time: these snapshots represent the particles displacements (relative to their equilibrium positions in the center-of-mass system) for a section of the chain, as a function of time, before and after applying the external force, for strong- and weak-friction representative cases. Each line in those snapshots represents the displacement of one particle, but the distance between lines was set to $0.075 r_{0}$, which is comparable to the maximum displacement, in order to amplify the deformations of the adsorbate. With snapshots of the actual positions of the particles it is very difficult to appreciate such distortions. Figures 9(a) and 9(b) correspond to a weakfriction case $(a / b=0.75)$, before and after the force is applied, respectively. Figures 9(c) and 9(d) are the analogous snapshots for a strong-friction case $(a / b=0.95)$. Comparing Figs. 9(a) and 9(c) there is an obvious difference between the structure of the chain in equilibrium (without external force), which is due to the constraint imposed by the substrate potential. In the strong case [Fig. 9(c)] the commensuration ratio is such that the system has to significantly rearrange to accommodate to the substrate potential, which is reflected in the deformations that can be observed and which yield a stable pattern. This in turn precludes the system vibrations in a specific collective mode, thus acting as a barrier for the propagation of specific frequencies (as if some modes are pinned) generating the minimum in the density of states of Fig. 8(b). This feature is absent in the snapshot of Fig. 9(a) which can hardly be distinguished from a free chain. So $\mathcal{D}(\omega)$ looks like a $1 \mathrm{D}$ case. When the external force sets the system in sliding motion the adlayer deformation acts as an excitation, forcing the system to vibrate in what was a forbidden mode for $F=0$. The frozen mode now does slide, as can be seen in Fig. 9(d), where the propagation of the pattern is quite apparent. Therefore, a strong absorption band appears precisely where there was a minimum in the force-free situation [Fig. 8(b)]. The corresponding weak-friction case shows no propagating pattern, and the whole structure is less perturbed [Fig. 8(a)]. However, the excitation of all the particles is evident, as for a free chain.

In conclusion, it is our understanding that this could explain the large differences in sliding friction behavior for different commensuration ratios: the key element is the appearance of a new channel of dissipation, due to a large wavelength deformation, that is responsible for the increased friction in the strong-friction cases. Such deformation appears when the adsorbate is near perfect commensuration with the substrate. This channel acts in parallel with the normal phonon dissipation mechanism.

The present results are consistent with those obtained by Braun et al. ${ }^{14}$ who, using molecular dynamics simulations, studied the mobility and diffusivity of a generalized FrenkelKontorova model taking into account anharmonic interactions. In Fig. 10 we compare our results with those of Braun et al. From the definition of mobility it is possible to write (a)

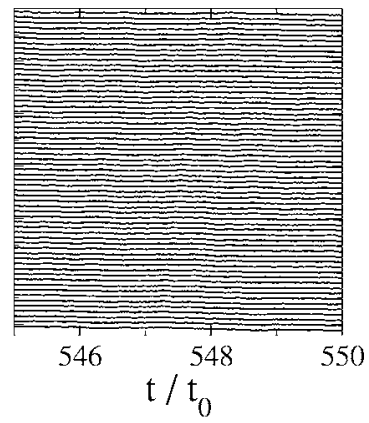

(b)

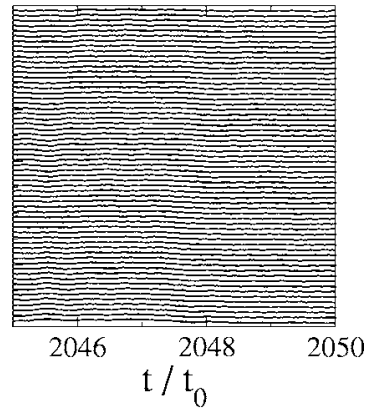

(c)

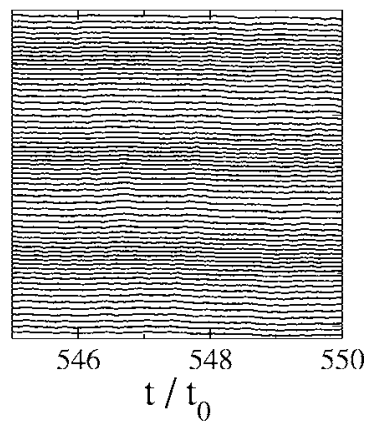

(d)

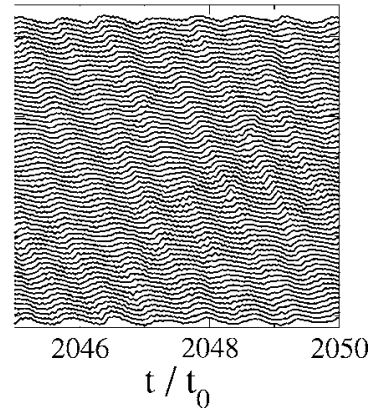

FIG. 9. Snapshots of the displacements of the particles as function of time (in units of $t_{0}=r_{0} \sqrt{\mathrm{m} / \varepsilon}$ ) relative to their equilibrium position in the center-of-mass system. In order to emphasize visualization of the structural change we have diminished the distances between particles down to $0.075 r_{0}$. Snapshots (a) and (b) correspond to a weak-friction case $(a / b=0.75)$, while snapshots (c) and (d) correspond to a strong-friction case $(a / b=0.95)$; snapshots (a) and (c) were taken before the application of force, while (b) and (d) were taken after that.

$$
B \propto \frac{1}{\eta} \propto \frac{1}{c},
$$

where $c$ is the proportionality coefficient of Eq. (8). Notice that the plots of Braun et al. have a maximum for the mo- 


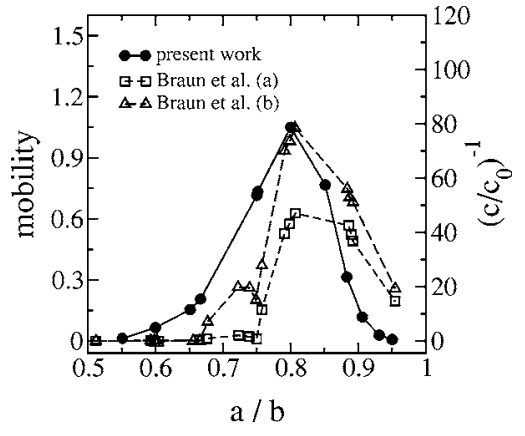

FIG. 10. Comparison between our results for the $c$ parameter and the data of Braun et al. of mobility as a function of commensuration ratio. Present work results are at $k_{B} T=0.05 \varepsilon(\approx 1 \mathrm{meV}$ for $\mathrm{Xe}$ ), while the results of Braun et al. are at $k_{B} T=2.5 \mathrm{meV}$ (a) and $k_{B} T=5 \mathrm{meV}$ (b). Curves (a) and (b) of Braun et al. are curves (1) and (2), respectively, of Fig. 2 from Ref. 14. $c_{0}=\left(m r_{0}^{2} \varepsilon^{3}\right)^{-1 / 2}$ and mobility is defined as $m \eta_{e l} v / F$. The scale was adjusted for the comparison between them.

bility (minimum for the friction coefficient) in the vicinity of our own maximum, in spite of the different potential and temperature used in our simulations. All of the latter points toward the robustness of our interpretation of the enhanced phononic friction.

\section{CONCLUSIONS}

A phononic friction coefficient calculation for a onedimensional model of an adsorbate-substrate interface has been presented. Using molecular dynamics simulations we investigated the system, for different substrate/adsorbate commensuration ratios $a / b$, in order to address two basic questions: first, how the phononic friction (and therefore the sliding friction) depends on the commensuration ratio between substrate and adsorbate; and second, whether a reasonable picture can be found to explain the divergent results between equivalent calculations in the literature. ${ }^{3-5} \mathrm{We}$ believe that both these goals have been attained since, for specific ranges of $a / b$ values, our model calculations yield a relatively large friction coefficient as compared with the intrinsic or electronic friction $\eta_{e}$. This picture holds if $a / b$ falls in what we denominate the strong-friction region; otherwise, $\eta_{p h}$ can be neglected in comparison with the electronic friction $\eta_{e}$.

From the technical point of view such sensitive dependence provides a plausible explanation for the divergent results found in the literature, especially for nearcommensurate systems like Xe-Ag. ${ }^{3-5}$ In fact, a small rotation of the adsorbate relative to the substrate in $2 \mathrm{D}$ or $3 \mathrm{D}$ simulations of sliding friction, can change the commensuration ratio between the surfaces in such a way as to generate a large change in effective friction, allowing one group to claim that electronic friction is dominant while the other states the opposite, both of them on the basis of properly carried out computations. Our conclusion is that simulations with much larger systems, and averaged over different realizations, have to be performed in order to minimize the artifact imposed by small system simulations or poor averaging.
This suggestion implies a rather formidable computational challenge, but certainly is more feasible than it was five or seven years ago.

Our results were obtained using classical molecular dynamics which is questionable when the temperature drops too far below the Debye temperature, because one may expect that the phonon contribution to dissipation, represented by Fig. 6, should drop rapidly due to quantum effects near that temperature, bringing the phonon contribution down toward the electronic term. However, as there is no reliable method for evaluating such an effect, it is still interesting to have classical simulation results down to nearly $T=0$, even when they cannot be directly compared to experiments. On the other hand, previous calculations ${ }^{4,5}$ for Xe on Ag were carried out at higher temperatures than the largest one in this contribution. At these temperatures the phononic friction is much larger and the sensitivity to $a / b$ effects decreases as $T$ rises.

Before arriving to the end we want to share some thoughts on dimensionality. The results presented here were obtained with a $1 \mathrm{D}$ model, because our aim was to address the topological influence of the substrate-adlayer relation on the resulting sliding friction, and we know from previous contributions in 3D systems that the substrate-adlayer commensuration ratio is difficult to control, due to possible — and maybe unavoidable - rotations of the adlayer. Even though the present results cannot be directly applied for a 2D or 3D system, we think that the Van Hove-type singularities can give a plausible explanation for the dramatic increase in friction for a 1D or a higher-dimensional system as well, when close to low-order commensuration $(a / b$ $\approx 0.5$ or 1 ) although maybe in a less impressive manner in 3D systems. On the other hand, one-dimensional "real" systems (done by deposition) with $a / b=0.5$, for example, do not show long-range order at finite temperature, but a mixture of domains, where atoms sit in the even or in the odd valleys, respectively. This implies the presence of defects or kinks at the domain boundary which are otherwise absent, since we are dealing with an infinite linear chain. Such kinks should have a strong influence on the friction properties, including pinning effects which would increase the threshold force. Again, in a real 2D or 3D system, due to possible relaxation mechanisms, such kinks should have less relevant consequences.

The present results might appear conflictive when compared with recent results on the friction of a dimer sliding on a periodic substrate. ${ }^{15,16}$ For the latter system the friction due to vibrations is maximum for a commensuration ratio $a / b$ $=2 / 3$. However, such a friction is due to resonance of the internal oscillation of the dimer that happens at much higher sliding velocities than the ones used here, since our purpose is to compare to typical experimental setups.

A final question can now be raised: Is the friction measured in the laboratory sensitive to these subtle changes in the topology, as seen by the sliding layer? One way to check on this would be to try different adsorbates on the same substrate (for example, comparing $\mathrm{Xe}, \mathrm{Kr}$, and Ar sliding on $\mathrm{Au}$ ) or the same adsorbate sliding over different substrates (Xe on $\mathrm{Au}$ or $\mathrm{Ag}$ ). 


\section{ACKNOWLEDGMENTS}

E.S.T. acknowledges the support of CNPq and CAPES. S.G. acknowledges hospitality of the Department of Physics and Astronomy of the University of New Mexico and support of the National Science Foundation under Grant
Nos. INT-0336343 and DMR-0097204, during the final stages of this work. M.K. was supported by FONDECyT Grant No. 1030957. This work was supported in part by Fundación Andes. We acknowledge the Linux cluster computational facility at HPC@UNM during the last stage of this work.
*Electronic address: esalcedo@if.ufrgs.br

†Electronic address: sgonc@if.ufrgs.br

FElectronic address: cscherer@if.ufrgs.br

${ }^{\S}$ Electronic address: mkiwi@puc.cl

${ }^{1}$ J. Krim and A. Widom, Phys. Rev. B 38, 12184 (1988).

${ }^{2}$ E. D. Smith, M. O. Robbins, and M. Cieplak, Phys. Rev. B 54, 8252 (1996).

${ }^{3}$ B. N. Persson and A. Nitzan, Surf. Sci. 367, 261 (1996).

${ }^{4}$ M. S. Tomassone, J. B. Sokoloff, A. Widom, and J. Krim, Phys. Rev. Lett. 79, 4798 (1997).

${ }^{5}$ A. Liebsch, S. Gonçalves, and M. Kiwi, Phys. Rev. B 60, 5034 (1999).

${ }^{6}$ M. O. Robbins and M. H. Müser, in Modern Tribology Handbook, edited by B. Bhushan (CRC Press, Boca Raton, 2001), pp. 717-765, and references cited therein.

${ }^{7}$ M. H. Müser, L. Wenning, and M. O. Robbins, Phys. Rev. Lett. 86, 1295 (2001).

${ }^{8}$ C. Daly and J. Krim, Phys. Rev. Lett. 76, 803 (1996).
${ }^{9}$ A. Dayo, W. Alnasrallah, and J. Krim, Phys. Rev. Lett. 80, 1690 (1998).

${ }^{10}$ M. P. Allen, Mol. Phys. 40, 1073 (1980).

${ }^{11}$ M. P. Allen, Mol. Phys. 47, 599 (1982).

${ }^{12}$ D. Frenkel and B. Smit, Understanding Molecular Simulation (Academic Press, San Diego, 1996).

${ }^{13}$ J. M. Dickey and A. Paskin, Phys. Rev. 188, 1407 (1969).

${ }^{14}$ O. M. Braun, T. Dauxois, M. V. Paliy, and M. Peyrard, Phys. Rev. B 54, 321 (1996).

${ }^{15}$ S. Gonçalves, V. M. Kenkre, and A. R. Bishop, Phys. Rev. B 70, 195415 (2004).

${ }^{16} \mathrm{~S}$. Gonçalves, C. Fusco, A. R. Bishop, and V. M. Kenkre, Phys. Rev. B 72, 195418 (2005).

${ }^{17}$ Forces in the range $(0.0001-0.001) \varepsilon / r_{0}$ are low in comparison with previous numerical works, but they are still very high in comparison with experimental setups; i.e., using parameters of $\mathrm{Xe}$, such forces produce velocities in the range of $4-20 \mathrm{~m} / \mathrm{s}$. 